\title{
Efeitos de um Manual de Instrução sobre o Repertório de Comportamentos de Acompanhantes de Crianças com Câncer
}

\author{
Izabel Cristina da Silva Brasiliense * \\ Inaê Duarte Benchaya \\ Eleonora Arnaud Pereira Ferreira \\ Universidade Federal do Pará, Belém, Pará, Brasil
}

\begin{abstract}
RESUMO
Este estudo teve por objetivo analisar os efeitos, em curto e em médio prazo, de um manual de instruções sobre comportamentos observados em acompanhantes e crianças com câncer durante realização de punção venosa em ambulatório. Participaram doze cuidadoras (idade média de 24 anos) de crianças cuja maioria $(n=8)$ tinha diagnóstico de leucemia. A coleta consistiu em uma observação antes da aplicação do manual e duas após o manual (efeito em curto e médio prazo). Observou-se diminuição significativa na taxa do comportamento de Desviar atenção do procedimento e uma tendência ao aumento na taxa do comportamento de Demonstrar carinho. Discute-se a efetividade do uso de manuais sobre mudanças comportamentais na área da saúde.
\end{abstract}

Palavras-chave: manual; comportamento; câncer.

\begin{abstract}
Effects of an instruction manual on behavioral repertoires of caregivers of children with cancer The aim of this study was to assess the short and mid-term effects of an instruction manual on the observable behaviors of caregivers of children with cancer during venipuncture in an outpatient setting. Twelve caregivers (mean age of 24 years old) participated while most children $(n=8)$ had been diagnosed with leukemia. Data collection consisted in one observation before, and two others after the manual presentation (short and mid - term effect). A significant decrease was observed in the rate of behavior of attention distraction from the procedure, and a tendency to an increase in showing love and affection. We discuss the effectiveness of the use of manuals on behavior changes in the area of health.
\end{abstract}

Keywords: manual; behavior; cancer.

$\mathrm{Na}$ área da saúde, manuais educativos podem ter, como um dos objetivos, aprimorar o conhecimento do paciente (e também do acompanhante, especialmente no caso de crianças e de idosos) sobre o tratamento e auxiliá-lo em seu autocuidado. Apresentam dicas sobre comportamentos saudáveis que favoreçam a autonomia e o controle sobre a situação vivenciada tanto pelo paciente quanto pelo acompanhante (Crepaldi, Rabuske, \& Gabarra, 2006; Echer, 2005; Ferreira, 2006; Moreira, Nóbrega, \& Silva, 2003; Oliveira, Fernandes, \& Sawada, 2008; McPherson, Higginson, \& Hearn, 2001; Pierce, 2010; Salles, \& Castro, 2010; Torres, Candido, Alexandre, \& Pereira, 2009).

Entretanto, existem algumas variáveis que devem ser consideradas na elaboração e avaliação de manuais.
Dentre elas, há o nível educacional do usuário. A recomendação, neste caso, é que a linguagem seja acessível e compreensível ao público alvo para que possa ocorrer aprendizagem (Brandt, McCree, Lindley, Sharp, \& Hutto, 2005; Castro, Pilger, Fuchs, \& Ferreira, 2007; Echer, 2005; Pierce, 2010; Salles, \& Castro, 2010; Torres et al., 2009; Vasconcellos-Silva, Rivera, \& Rozemberg, 2003).

Outra variável que pode influenciar sobre os efeitos do manual diz respeito às autorregras instaladas no repertório comportamental do usuário antes da aplicação deste instrumento. Autorregras consistem em regras formuladas ou reformuladas pelo próprio indivíduo que passa a se comportar a partir do controle exercido por elas. São parte do repertório comporta- 
mental verbal que afeta outra parte do repertório (seja verbal ou não verbal) do indivíduo (Jonas, 1997).

No caso de acompanhantes de pacientes pediátricos, outra variável que também pode influenciar os efeitos do manual é o tempo em tratamento do paciente. No caso de crianças com câncer, Kohlsdorf e Costa Jr. (2008) apontam que há mudanças comportamentais devido ao tratamento médico ser prolongado, com repetidas exposições a procedimentos invasivos, além da própria internação hospitalar e dos efeitos colaterais do tratamento. Neste sentido, estudos têm apontado mudanças nas estratégias de enfrentamento utilizadas por acompanhantes de crianças com câncer ao longo do tempo (Dolgin et al., 2007; HoekstraWeebers, Heuvel, Jaspers, Kamps, \& Klip, 1998; Kohlsdorf, \& Costa Jr., 2009).

Estratégias de enfrentamento (tradução do termo coping) correspondem ao repertório comportamental utilizado para adaptar-se a situações de ameaça iminente ou de desafio, como no caso de doenças e tratamentos (Kohlsdorf, \& Costa Jr., 2008). No estudo de Kohlsdorf e Costa Jr. (2009), foi observado que, no início do tratamento, os acompanhantes de crianças com câncer utilizaram mais frequentemente estratégias focalizadas na emoção (esquiva e fuga, distração etc.) e, ao longo do tempo de tratamento, passaram a utilizar estratégias focalizadas no problema (buscar informações sobre a doença, controle da medicação etc.).

O estudo de Beltrão, Vasconcelos, Pontes e Albuquerque (2007) menciona que, no caso de crianças com câncer, por demandarem tratamento longo e em ambiente hospitalar, os acompanhantes passam a utilizar a presença de outros acompanhantes como estratégia de enfrentamento focalizada no apoio social. Nesse contexto, trocam experiências sobre as contingências semelhantes às quais estão expostos, ficando, portanto, sob o controle de outras variáveis além do uso de manual de instruções.

Segundo Moreira et al. (2003), é imprescindível realizar a avaliação dos manuais que são elaborados e utilizados pelos profissionais de saúde. No entanto, por meio de uso exclusivo de questionários só é possível ter acesso a autorrelatos, não havendo garantia de avaliar a efetiva ocorrência de aprendizagem e mudança de comportamento nos usuários destes ma- nuais. Desse modo, haveria a necessidade de se obter também informações a partir da observação direta do comportamento alvo.

O estudo de revisão da literatura realizado por Vasconcellos-Silva et al. (2003) fez críticas à elaboração de manuais sem que haja dados substanciais da mudança de comportamento subsequente. Estes autores sugerem que manuais construídos em conjunto com os usuários, a partir de uma interação ativa, apresentaram dados mais consistentes de mudança.

Ainda são poucos os estudos que têm apontado para mudança de comportamentos a partir da utilização de manuais. Em um destes estudos, MacFarlane, Holmes e MacFarlane (1997) relataram que foi possível reduzir o número de consultas desnecessárias feitas por pacientes com doença crônica, a partir do uso de manual. Em outro estudo, Syrjala et al. (2008) elaboraram um manual com vídeo sobre dor e seu tratamento e obtiveram nas reavaliações que, em comparação com o grupo controle, houve redução na percepção de dor e aumento na adesão ao tratamento no grupo submetido ao manual e vídeo. Tanto no primeiro quanto no segundo estudo, os autores utilizaram grupo controle e análises estatísticas para comparar os dados dos grupos. No entanto, no segundo estudo, no qual foram feitas várias medidas de pós-teste, não foi possível verificar os efeitos em separado do manual e vídeo.

Manuais têm sido muito utilizados em vários contextos da área da saúde, dentre eles o de tratamento do câncer em crianças (Duarte, 2012; Ferreira, 2005; Salles, \& Castro, 2010). No entanto, apenas um dos estudos encontrados nesta área, o de Duarte (2012), analisou manuais para acompanhantes de crianças com câncer por meio de observação direta a fim de se obter uma medida comportamental dos efeitos destes instrumentos. Houve obtenção, em sua maioria, de dados apenas pelo relato oral dos acompanhantes (Ferreira, 2005; Salles, \& Castro, 2010). O mesmo foi encontrado em outros estudos que avaliaram diversos manuais, apenas a partir do relato verbal, como o de Fonseca, Scochi, Rocha e Leite (2004), Grippo e Fracolli (2008), Reberte, Hoga e Gomes (2012) e Silva e Cardoso (2009).

O único estudo encontrado que fez avaliação por meio de observação direta do comportamento foi o de 
Duarte (2012), o qual comparou o efeito do uso de um manual de instruções sobre quimioterapia e punção venosa com o efeito do uso de um treino parental realizado com acompanhantes de crianças com câncer. Os resultados apontaram que, na Condição Rotina, os participantes (cuidadores) não variaram seus comportamentos ao longo das sessões, com dois participantes mantendo alta frequência de comportamentos negligentes e um participante mantendo monitoria positiva. $\mathrm{Na}$ Condição Manual, observou-se mudança de comportamento em dois cuidadores como efeito imediato à utilização do manual, mas não em longo prazo. Na Condição 3, na qual os cuidadores foram submetidos ao Protocolo de treino parental, observou-se aumento na frequência de monitoria positiva em curto e longo prazo. Para os participantes da Condição Manual, verificou-se mudança em curto prazo no comportamento dos acompanhantes, principalmente em relação à Monitoria Positiva. Entretanto, não foi observada a manutenção desse efeito após a realização de uma terceira sessão de observação direta depois de no mínimo uma semana da segunda sessão. Este estudo foi realizado com apenas três díades (acompanhantecriança) em cada condição e não foram feitas análises estatísticas para verificar se o efeito observado, mesmo que em curto prazo, foi significativo.

A partir do exposto, o presente estudo teve por objetivo analisar os efeitos, em curto e em médio prazo, de um manual de instruções sobre comportamentos observados em acompanhantes e crianças com câncer durante realização de punção venosa em ambulatório.

\section{MÉTODO}

\section{Participantes}

Participaram doze crianças (seis meninas e seis meninos) e seus acompanhantes, atendidos no ambulatório para quimioterapia do serviço de oncologia pediátrica de um hospital de referência na cidade de Belém, PA.

Os participantes foram selecionados a partir dos seguintes critérios: (a) a criança deveria ter idade entre 2 a 11 anos, ter diagnóstico de câncer com indicação de tratamento quimioterápico e já ter sido submetida a pelo menos uma sessão de quimioterapia em ambulatório; (b) o acompanhante deveria ter idade igual ou superior a 18 anos, concordar em participar da pesqui- sa e assinar o Termo de Consentimento Livre e Esclarecido (TCLE).

As crianças selecionadas tinham entre dois e nove anos de idade $(M=4 \pm 1)$. A maioria apresentava diagnóstico de leucemia $(n=8)$. O período médio de tratamento das crianças era de três meses ( $\mathrm{DP}=2)$, no momento de ingresso na pesquisa. Sete estavam há menos de três meses em tratamento e cinco estavam há mais de três meses.

Os acompanhantes selecionados eram todos do sexo feminino e tinham idade entre 18 e 31 anos $(M=24$ \pm 4 ). A maioria das acompanhantes tinha escolaridade correspondente ao Ensino Fundamental $(n=7)$ e Ensino Médio $(n=5)$, residia em outro município $(n=9)$ e era da classe econômica $\mathrm{C} 2(\mathrm{n}=6)$ segundo os critérios da Associação Brasileira de Empresas de Pesquisa [ABEP] (2008).

\section{Instrumentos}

(1) Roteiro de entrevista semiestruturado: elaborado para este estudo com o objetivo de coletar dados acerca de características sociodemográficas dos participantes, incluindo o Critério de Classificação Econômica Brasil (ABEP, 2008);

(2) Manual de orientação sobre quimioterapia e punção venosa (Brasiliense, Duarte, \& Ferreira, 2013): elaborado para esta pesquisa, tinha o objetivo de instruir cuidadores de crianças com câncer em tratamento quimioterápico com informações acerca do que é quimioterapia e de como é feita a punção venosa; ainda, continha instruções e sugestões, exemplificadas por meio de figuras, de dicas comportamentais sobre como lidar com as crianças durante a punção venosa, considerando três eixos (carinho/suporte, negligência/agressão e atenção/distração);

(3) Escala de Avaliação do Manual: adaptada de Lima (2009), esta escala foi utilizada com o objetivo de verificar se as instruções fornecidas pelo manual aumentaram o conhecimento do acompanhante sobre punção venosa e verificar se o acompanhante tinha dúvidas sobre o conteúdo do manual. Esta escala consistiu em seis itens sobre o manual medidos em frequência de comportamento autorrelatado, com uma escala tipo Likert com 5 pontos, variando de 1 (discordo totalmente) a 5 (concordo totalmente). Os cinco itens eram: (1) obteve esclarecimento das dúvidas 
quanto à punção venosa, (2) sentiu-se tranquila em relação ao procedimento da criança, (3) sentiu-se preocupada quanto à forma de se comportar durante a punção venosa, (4) as informações ajudaram a lidar com o procedimento da criança, (5) teve dificuldade para compreender algum item do manual;

(4) Escala de Observação Direta do Comportamento (Observation Scale of Behavior Distress [OSBD]): adaptada no Brasil por Borges (1999) e Costa Jr. (2001), apresenta categorias morfológicas de comportamentos que englobam respostas verbais, vocais e motoras da criança, as quais sinalizam experiências indicativas de sofrimento ou não em situações de submissão a procedimentos médicos invasivos. Para este estudo, as categorias foram adaptadas de acordo com os dados obtidos, tendo como base as categorias prévias criadas por Costa Jr. (2001), mantendo-se a classificação dos comportamentos em não concorrentes (os comportamentos que facilitam ou não dificultam a realização do procedimento) e em concorrentes (aqueles que atrasam, dificultam ou impedem a realização do procedimento). Para os acompanhantes, foram utilizadas 27 categorias comportamentais que também englobam respostas verbais, vocais e motoras e que também foram adaptadas para este estudo, classificadas em manter atenção ao procedimento, demonstrar carinho, conter e desviar atenção.

\section{Procedimento}

Após aprovação do projeto pelo Comitê de Ética em Pesquisa envolvendo seres humanos, o estudo foi realizado em duas etapas: elaboração do manual e avaliação dos efeitos do manual por meio de observação direta do comportamento.

\section{Elaboração do manual}

Inicialmente, fez-se uma revisão da literatura sobre modelos de manuais para orientação em oncologia pediátrica (Ferreira, 2005; Lima, 2009; Salles, \& Castro, 2010). Em seguida, fez-se a observação direta da rotina do ambulatório de quimioterapia do hospital onde o estudo seria realizado. Nesta ocasião, fez-se o registro dos comportamentos emitidos por 18 crianças e seus acompanhantes (após consentimento livre e esclarecido) durante a realização de uma punção venosa para quimioterapia, com o objetivo de treinar a pesquisadora e uma auxiliar de pesquisa nos instru- mentos de coleta de dados. Em seguida, fez-se o teste de fidedignidade entre as duas observadoras, obtendose concordância de até $80 \%$ entre as categorias de comportamento selecionadas para análise.

Com base nestas informações, foi construída a primeira versão do Manual de orientação sobre quimioterapia e punção venosa para acompanhantes de pacientes pediátricos em tratamento quimioterápico ambulatorial.

Uma cópia da primeira versão foi entregue a profissionais especialistas da área de oncologia, incluindo três médicos e seis enfermeiros da equipe de Oncologia Pediátrica do hospital selecionado, a fim de confirmar e/ou retificar as informações contidas no manual. A partir das sugestões feitas por estes profissionais, fez-se uma segunda versão do manual, agora contendo a apresentação de ilustrações/figuras com dicas comportamentais.

Em seguida, uma cópia da segunda versão com as alterações sugeridas pelos profissionais de saúde foi entregue e lida com outros cinco acompanhantes de crianças em tratamento quimioterápico, com o objetivo de obter-se a avaliação do manual sob o ponto de vista do usuário. Por fim, uma cópia da terceira versão do manual foi entregue a um especialista em Psicooncologia pediátrica com história de elaboração e de utilização de manuais de orientação em saúde, cujas sugestões permitiram a elaboração da versão final do manual (Brasiliense et al., 2013).

\section{Avaliação dos efeitos do manual}

Após o treino das pesquisadoras e elaboração do manual, deu-se início à coleta de dados. Foram convidados a participar os acompanhantes que aguardavam, em sala de espera do ambulatório de quimioterapia do serviço de Oncologia Pediátrica do hospital selecionado, para realização da punção venosa com a criança. Explicava-se a cada acompanhante o objetivo da pesquisa e, em caso de anuência para participação, lia-se o TCLE. Em seguida, era observado o procedimento de punção venosa agendado para aquele dia.

Após a observação, foi realizada uma entrevista com a acompanhante a fim de entregar e fazer a leitura explicativa do Manual. Na sessão seguinte de quimioterapia, que poderia ocorrer em média uma semana após a aplicação do manual, dependendo do tratamento quimioterápico da criança, fez-se uma segunda sessão de 
observação do procedimento de punção venosa, seguida da aplicação da Escala de Avaliação do Manual. Após um período mínimo de uma semana e máximo de quinze dias da segunda observação, foi realizada uma terceira observação do procedimento de punção.

Todas as sessões de observação foram registradas por meio de gravação em áudio, ditadas pelas pesquisadoras na sequência de ocorrência dos comportamentos da criança e do acompanhante, com base no sistema de categorias de análise adaptado de Costa Jr. (2001).

\section{ANÁLISE DOS DADOS}

Foram realizados três níveis de avaliação do Manual: avaliação pelo relato dos acompanhantes, avaliação do efeito em curto prazo e avaliação do efeito em médio prazo.

A avaliação pelo relato dos acompanhantes foi feita mediante a análise dos dados obtidos por meio da aplicação da Escala de avaliação do manual.

Para a avaliação do efeito em curto prazo, foram utilizadas as duas primeiras sessões de observação direta do comportamento do acompanhante e da criança durante a punção venosa para quimioterapia. Os dados da primeira sessão foram considerados como linha de base e comparados com os dados da segunda sessão, realizada após a aplicação do manual com a acompanhante.

Para a análise do efeito do Manual em médio prazo, foram comparadas a segunda e terceira sessão de observação direta do comportamento do acompanhante e da criança durante o procedimento de punção venosa, utilizando-se novamente a Escala de Observação Direta do Comportamento. Optou-se pela comparação da segunda com a terceira sessão com o objetivo de avaliar a mudança e a manutenção dos comportamentos dos acompanhantes após a introdução do manual.

Os dados obtidos por meio das sessões de observação foram analisados a partir de dois sistemas de categorias de análise (comportamentos dos acompanhantes e comportamentos das crianças) descritos a seguir.

\section{Categorias de comportamentos dos acompanhantes}

Manter atenção ao procedimento (MAT): olhar em direção ao procedimento, auxiliar a enfermeira, infor- mar a criança sobre os passos do procedimento, falar positivamente e com incentivo à colaboração da criança.

Demonstrar carinho $(D E C)$ : permanecer próximo à criança, tocar na criança, abraçar, enxugar o rosto, sorrir para a criança, acariciá-la, segurar a mão e beijar.

Conter (CON): utilizar os braços ou pernas para impossibilitar, à força, qualquer movimentação da criança, mantendo-a em uma mesma posição a fim de permitir acesso à área onde será realizada a punção por parte do técnico em enfermagem.

Desviar atenção (DAT): obstruir visão da criança, explorar ambiente, orientar o olhar em direção a objetos ou pessoas presentes no local em que está sendo realizado o procedimento, mantendo sua atenção distante da criança e se manter afastada da criança.

\section{Categorias de comportamentos das crianças}

Comportamentos não concorrentes $(\mathrm{CNC})$ : comportamentos que facilitam ou não dificultam a realização do procedimento de punção venosa pela enfermeira, como buscar suporte emocional da acompanhante (p. ex., elevar o braço em direção e segurar parte do corpo - braço, barriga etc. - do acompanhante), olhar para o procedimento que está sendo executado, sinalizar dor por meio de expressões faciais, falar (com acompanhante ou técnico em enfermagem) e explorar ambiente (orientar o olhar em direção a objetos ou pessoas presentes no local em que está sendo realizado o procedimento).

Comportamentos concorrentes (COC): comportamentos que atrasam, dificultam ou impedem a realização do procedimento de punção venosa pela enfermeira, como chorar, choramingar, comportar-se de modo nervoso (p. ex., sacudir o braço ou as pernas), gritar e protestar (p. ex., negar-se a realizar a punção; puxar o braço em direção ao próprio corpo).

Para a análise dos comportamentos dos acompanhantes e dos comportamentos das crianças, inicialmente fez-se a transcrição das gravações em áudio e foi construído um banco de dados com base na ocorrência de cada categoria de comportamento registrada em cada sessão de observação. Em seguida, a análise foi realizada por meio do cálculo da taxa de cada categoria de comportamento, dividindo-se o total de ocorrências pelo tempo de cada sessão de observação de punção venosa. Depois, fez-se o cálculo das médias 
por categoria de comportamento, o que permitiu a análise dos efeitos de curto e médio prazo. Para a análise estatística foi utilizado o Teste dos postos com sinais de Wilcoxon, a fim de verificar se havia diferenças estatisticamente significativas entre os dados por sessão. Também foi utilizado o coeficiente de correlação de Sperman, para verificar se haviam associações entre as categorias comportamentais e as variáveis: tempo em tratamento da criança e nível de escolaridade dos acompanhantes.

\section{RESULTADOS}

\section{Avaliação do Manual pelo relato dos acompanhantes}

A frequência absoluta (n) e a frequência relativa (\%) das respostas das acompanhantes à Escala de avaliação do Manual estão apresentadas na Tabela 1 .

A partir dos dados da Tabela 1, pôde-se observar que quase a totalidade das acompanhantes afirmou que as informações contidas no Manual puderam esclarecer suas dúvidas sobre punção venosa e sobre como se comportar durante o procedimento, deixando-as tranquilas, o que facilitaria o manejo com a criança durante o procedimento.

Tabela 1

Frequência absoluta e relativa das respostas das acompanhantes à Escala de avaliação do manual

\begin{tabular}{|c|c|c|c|}
\hline Tópicos & $N$ & $\%$ & Exemplo de relato \\
\hline Dúvidas esclarecidas & 12 & 100 & $\begin{array}{l}\text { "Esclareceram... pois, não entendia nada, agora sei mais [...] agora tento } \\
\text { participar o máximo que posso, antes deixava tudo com a enfermeira". }\end{array}$ \\
\hline $\begin{array}{l}\text { Tranquilidade em relação ao } \\
\text { procedimento }\end{array}$ & 12 & 100 & "A mãe saber como tratar o filho [...] ela se tranquiliza mais". \\
\hline $\begin{array}{l}\text { Sem preocupação quanto à ma- } \\
\text { neira de se comportar durante a } \\
\text { punção venosa }\end{array}$ & 11 & 91,6 & $\begin{array}{l}\text { "Aprendi a lidar com a situação [...] ficou mais fácil". } \\
\text { "Tinha o costume de prender ela e descobri que faz mal". }\end{array}$ \\
\hline $\begin{array}{l}\text { Ajudou a lidar com o procedi- } \\
\text { mento }\end{array}$ & 12 & 100 & $\begin{array}{l}\text { "Agora não só converso com ele, mas tento manter ele próximo colocando } \\
\text { no colo, tento seguir o que li". }\end{array}$ \\
\hline $\begin{array}{l}\text { Sem dificuldade para compreen- } \\
\text { der o conteúdo do manual }\end{array}$ & 12 & 100 & "[O Manual] Explica bem as coisas". \\
\hline
\end{tabular}

Fonte: Protocolo de pesquisa.

\section{Avaliação dos efeitos do Manual em curto e em médio prazo}

As sessões de punção venosa tiveram, em média, duração de quatro minutos, tanto para a primeira (amplitude de um a dez minutos) quanto para a segunda sessão de observação (amplitude de dois a dez minutos), e de seis minutos para a terceira sessão (amplitude de quatro a quinze minutos). As sessões tiveram, em sua maioria, uma única tentativa de punção venosa para todas as crianças participantes $(n=27$ de 36 sessões).

\section{(a) Comportamentos observados nas acompanhantes}

Na Figura 1, estão as médias das taxas de ocorrência dos comportamentos de Manter Atenção (MAT), Demonstrar carinho (DEC), Conter (CON) e Desviar atenção (DAT), observadas nas acompanhantes nas três sessões de punção venosa realizadas com a criança. 


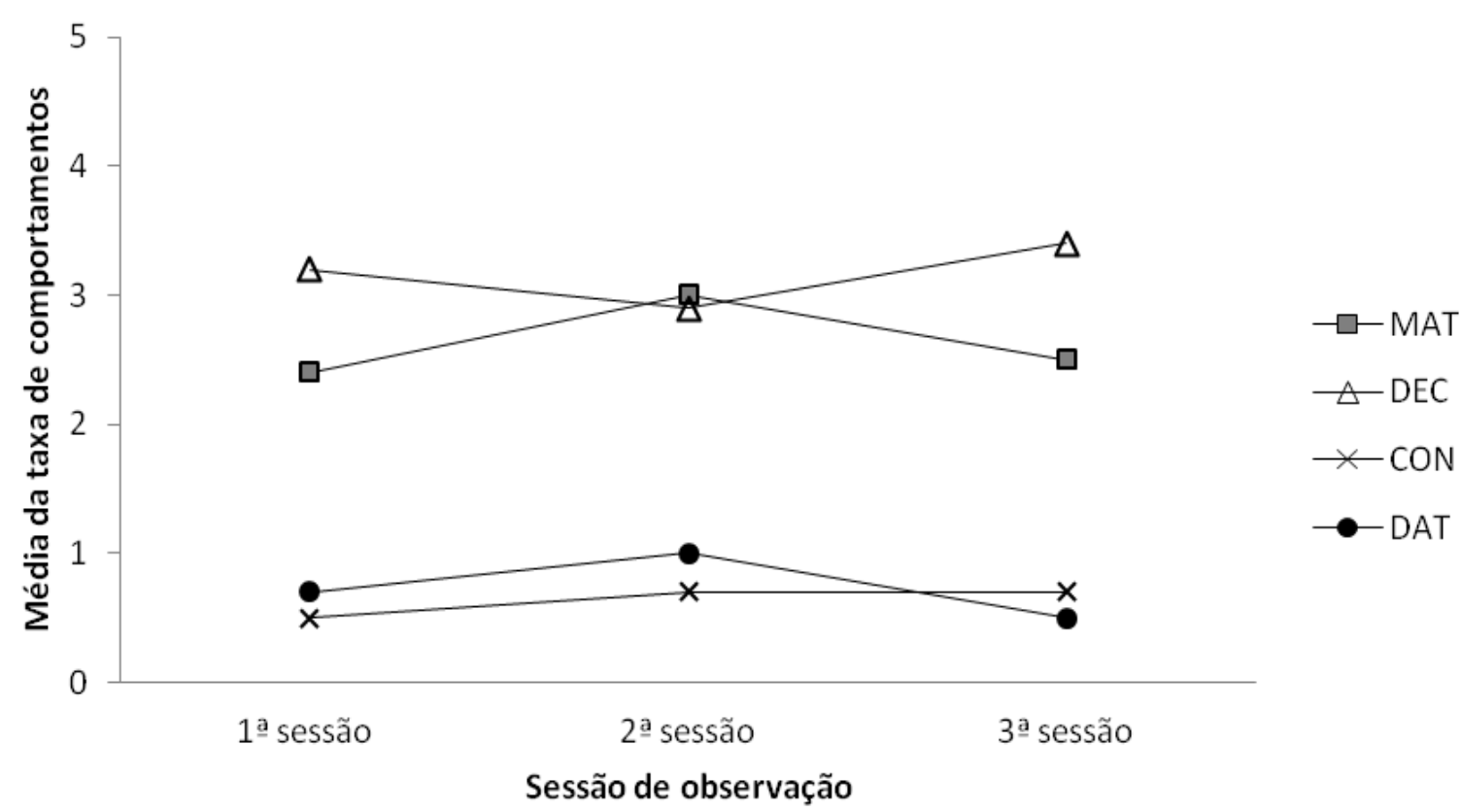

Figura 1. Média das taxas dos comportamentos de Manter atenção (MAT), Demonstrar carinho (DEC), Conter (CON) e Desviar Atenção (DAT) obtida pelas acompanhantes ao longo das três sessões de observação.

Ao comparar as taxas dos comportamentos emitidos pelas acompanhantes em cada sessão de observação, verificou-se que, na primeira sessão (anterior ao uso do Manual), as acompanhantes apresentaram maior taxa de comportamentos de DEC (taxa igual a 3,2) seguido de MAT $(2,4)$. As menores taxas foram as de CON $(0,5)$ e de DAT $(0,7)$.

Para análise dos efeitos em curto prazo, verificou-se que não houve diferença estatisticamente significativa entre a primeira e segunda sessão de observação para nenhuma das categorias de comportamento avaliadas.

Para a análise do efeito prolongado, verificou-se que houve diferença significativa apenas na categoria DAT $(\mathrm{T}=3 ; p=0,03 ; r=0,4)$. Este resultado indica que a taxa do comportamento de Desviar Atenção foi significativamente mais baixa na terceira sessão ( $M=$ $0,5)$ do que na segunda $(M=1,0)$, com tamanho de efeito moderado na classificação de Cohen.

Com relação à categoria Demonstrar Carinho, embora a diferença entre a terceira e a segunda sessão de observação não tenha sido considerada estatisticamente significativa $(p=0,06)$, pode-se dizer que houve uma tendência ao aumento na taxa deste comportamento da segunda $(M=2,9)$ para a terceira sessão $(M=$ $3,4)$ de observação.
Fez-se análise estatística para verificar se o tempo de tratamento das crianças era uma variável relevante para o seguimento das instruções contidas no Manual pelas acompanhantes, considerando-se características de inexperiência destas à situação e o caráter mais agressivo no início do tratamento das crianças.

No que diz respeito ao tempo de tratamento, não houve correlações significativas. Contudo, houve uma tendência à associação inversa entre o tempo de tratamento e o comportamento de Demonstrar Carinho $(\rho=-0,51 ; p=0,09)$, sugerindo taxas mais baixas deste comportamento nas acompanhantes cujas crianças estavam há mais tempo em tratamento.

Com relação à escolaridade, não houve correlações significativas, indicando que o nível educacional não influenciou as taxas de emissão dos comportamentos avaliados.

\section{(b) Comportamentos observados nas crianças}

As médias das taxas de ocorrência dos Comportamentos Concorrentes (COC) e dos Não concorrentes (CNC) observadas nas crianças estão dispostas na Figura 2. 


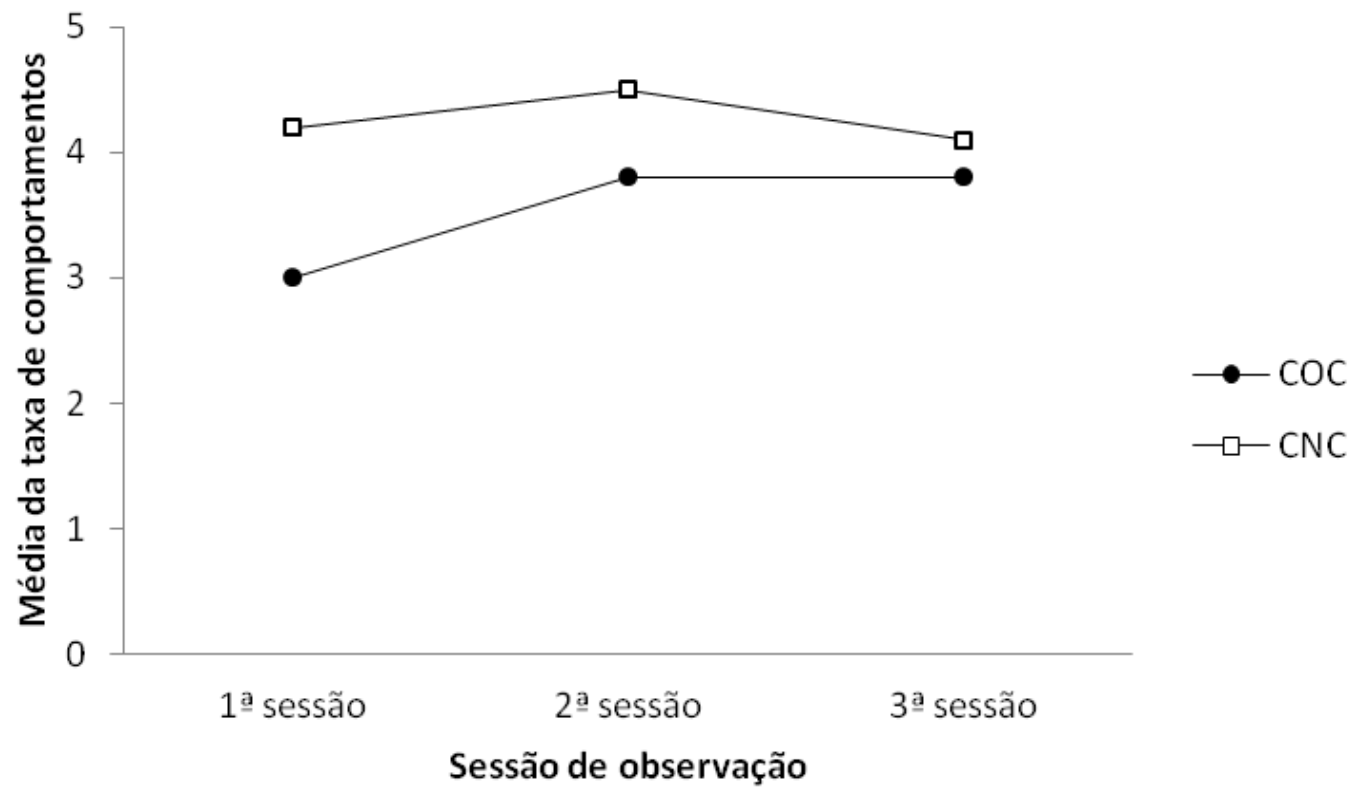

Figura 2. Média das taxas de ocorrência dos Comportamentos Concorrentes (COC) e dos Comportamentos Não Concorrentes $(\mathrm{CNC})$ obtidas pelas crianças ao longo de três sessões de observação direta do procedimento de punção venosa em ambulatório.

Ao comparar as taxas dos comportamentos emitidos pelas crianças por sessão de observação, verificou-se, em todas as sessões, maior taxa de CNC ao ser comparado com COC, na ordem de 4,2 na primeira sessão; 4,5 para a segunda e 4,1 para a terceira.

Para análise dos efeitos em curto prazo, os resultados indicam que não houve diferença estatisticamente significante entre a primeira e segunda sessão de observação, assim como da segunda para terceira sessão para ambas as categorias comportamentais COC e CNC observadas nas crianças.

Com relação aos comportamentos apresentados pelas crianças de acordo com o tempo em tratamento destas, verificou-se que não houve associação entre o tempo de tratamento e as categorias comportamentais COC e CNC.

\section{DISCUSSÃO}

O presente estudo se caracterizou por ser uma tentativa de avaliação, realizada por meio de observação direta do comportamento, dos efeitos de um manual educativo sobre comportamentos de acompanhantes de crianças com câncer. Os resultados diferem dos estudos de Salles e Castro (2010) e de Ferreira (2005), que apresentaram resultados obtidos somente por meio de relatos dos participantes.
Quanto aos efeitos do uso do Manual sobre o comportamento das acompanhantes, os principais resultados indicam que foi observada diminuição significativa na taxa do comportamento de Desviar atenção do procedimento, e uma tendência ao aumento na taxa do comportamento de Demostrar carinho. Tais resultados podem ser considerados como efeitos do uso do Manual.

Quanto aos demais comportamentos analisados, observou-se que não houve diferença estatisticamente significativa entre as sessões de observação. No entanto, houve um aumento na taxa de ocorrência da categoria Manter atenção (MAT) da primeira para a segunda sessão de observação, o que sugere que as acompanhantes podem ter ficado sob o controle das regras expostas no Manual que especificam tal comportamento. Ainda que esta diferença não tenha sido estatisticamente significante, indica ter ocorrido mudança comportamental a partir do uso do Manual para estas acompanhantes. Este resultado corrobora a crítica feita por Vasconcelos-Silva et al. (2003) à falta de dados obtidos a partir de observação direta que justifiquem o uso de manuais educativos quando o objetivo é mudança de comportamento.

De modo geral, as acompanhantes exibiram maior taxa de comportamentos de Demonstrar carinho e de Manter atenção ao procedimento nas três sessões, ao 
se comparar com os comportamentos de Conter e Desviar atenção. Uma possível explicação para tal resultado é o fato de as sessões de punção venosa terem sido realizadas em uma enfermaria na qual havia a presença de outras acompanhantes. Nestes casos, uma podia observar os comportamentos da outra no momento da punção venosa, podendo haver trocas de informações e experiências entre as mesmas, como apontado por Beltrão et al. (2007) quanto às acompanhantes serem expostas a contingências semelhantes e assim servirem de apoio social mútuo. Por outro lado, considerando-se que a maioria das participantes já estava, em média, há três meses acompanhando a rotina de tratamento, sugere-se que houve adaptação à situação de tratamento conforme sinalizado em Kohlsdorf e Costa Jr. (2008), Kohlsdorf e Costa Jr. (2009) e em Dolgin et al. (2007).

Verificou-se que houve uma tendência à associação inversa entre o tempo de tratamento e o comportamento de Demonstrar Carinho, ou seja, sugerindo taxas mais baixas deste comportamento naquelas acompanhantes cujas crianças estavam há mais tempo em tratamento. Tal resultado corrobora os do estudo de Kohlsdorf e Costa Jr. (2009) em que as estratégias de enfrentamento no início do tratamento têm maior foco na "emoção" por parte das acompanhantes.

Não foram encontradas associações entre os comportamentos das acompanhantes e o nível educacional, o que indica que o conteúdo do Manual estava acessível a todas as participantes do estudo. Esse fato provavelmente ocorreu devido à utilização de figuras com dicas comportamentais ao invés de exclusivamente textos explicando os comportamentos e também aos passos seguidos para sua elaboração. Esta é uma das recomendações feitas para a elaboração de manuais dirigidos à população em geral visando facilitar a aprendizagem, conforme sugerido por Echer (2005), Pierce (2010), Salles e Castro (2010) e Torres et al. (2009).

Quanto à diferença significativa observada na taxa do comportamento de Desviar Atenção do procedimento (DA), da segunda para a terceira sessão, considera-se que esta categoria poderia estar sob controle de autorregras do tipo "não há o que fazer durante o procedimento", conforme sugerido por Jonas (1997). Para exemplificar, conforme fora apresentado na Tabela 1, uma das participantes mencionou durante a entrevista que antes da leitura do Manual "deixava tudo com a enfermeira", e que após a leitura passou a participar mais ativamente do procedimento, dizendo: Agora não só converso com ele, mas tento manter ele próximo, colocando no colo, tento seguir o que li. $\mathrm{O}$ Manual, portanto, criou condições para que as acompanhantes avaliassem as contingências em vigor durante o procedimento de punção venosa, permitindo que deixassem de seguir a possível autorregra estabelecida, ao contrário do observado no estudo de Gomes (2010).

Quanto aos resultados obtidos por meio do relato das acompanhantes a partir da Escala de avaliação do manual, estes são semelhantes aos encontrados em estudos sobre avaliação do efeito de manuais de instruções. Em tais estudos, as acompanhantes mencionaram que o manual as ajudou a lidar melhor com a situação de tratamento e que não tiveram dificuldades para compreender as informações fornecidas (e.g. Ferreira, 2005; Fonseca et al., 2004; Grippo, \& Fracolli, 2008; McPherson et al., 2001; Reberte et al., 2012; Salles, \& Castro, 2010; Torres et al., 2009).

Em relação às crianças, não houve diferença significativa entre Comportamentos concorrentes e Comportamentos não concorrentes ao longo das três sessões de observação, o que também fora encontrado no estudo de Lemos e Ferreira (2010), o qual foi realizado com amostra semelhante à do presente estudo. Uma possível hipótese para maior taxa de comportamentos não concorrentes pode ter sido o fato de as acompanhantes já apresentarem em seu repertório comportamental maior taxa de Demonstrar carinho e de Manter atenção ao longo das sessões.

No estudo de Duarte (2012), no qual foi utilizado o mesmo Manual elaborado neste estudo, verificou-se efeito em curto prazo com mudança no comportamento das crianças em relação aos comportamentos concorrentes. Tais resultados se diferenciam do presente estudo, no qual não foram encontradas diferenças significativas entre os comportamentos das crianças, ao longo das três sessões de observação. No entanto, no estudo de Duarte (2012), não foram realizados testes estatísticos para analisar se a diferença dos comportamentos por sessão era significativa. No presente estudo, utilizou-se da média, pois se pretendeu investigar um instrumento utilizado pelo serviço de 
saúde para população em geral, que é construído para atingir o máximo de usuários, não sendo, portanto, individualizado.

$\mathrm{Na}$ literatura, têm-se relatado a avaliação do manual por meio do conhecimento adquirido pelas acompanhantes e/ou pacientes após a aplicação deste instrumento (Ferreira, 2005; Fonseca et al., 2004; Grippo, \& Fracolli, 2008; Reberte et al., 2012; Salles, \& Castro, 2010; Silva, \& Cardoso, 2009). No presente estudo, deu-se ênfase aos dados obtidos por meio de observação direta dos comportamentos das crianças e das acompanhantes. Sugere-se que estudos futuros abordem os dois tipos de avaliação (produção de conhecimento e mudança de comportamento) para que possam ser elaborados manuais mais eficazes na promoção da saúde.

Uma vez que o tratamento de câncer é prolongado e difícil, sugere-se também a realização de estudos longitudinais, avaliando a necessidade de manuais para essa população desde o início do tratamento.

\section{REFERÊNCIAS}

Associação Brasileira de Empresas de Pesquisa (2008). Critério de Classificação Econômica Brasil. Retirado de http://www.abep.org.br.

Beltrão, M. R. L. R., Vasconcelos, M. G. L., Pontes, C. M., \& Albuquerque, M. C. (2007). Câncer infantil: Percepções maternas e estratégias de enfrentamento frente ao diagnóstico. Jornal de Pediatria, 83(6), 562-566.

Borges, L. M. (1999). Treinamento de respostas de enfrentamento e colaboração em mães de crianças submetidas a procedimentos médicos invasivos (Dissertação de mestrado). Universidade de Brasília, Brasília.

Brandt, H. M., McCree, D. H., Lindley, L. L., Sharpe, P. A., \& Hutto, B. E. (2005). An evaluation of printed HPV education materials. Cancer Control Journal, 12(5), 103-106.

Brasiliense, I. C. S., Duarte, I. B., \& Ferreira, E. A. P. (2012). Punção venosa em crianças: Manual para acompanhantes. Belém: UFPA.

Castro, M. S., Pilger, D., Fuchs, F. D., \& Ferreira, M. B. C. (2007). Development and validity of a method for the evaluation of printed education material. Pharmacy Practice, 5(2), 89-94.

Costa Jr., A. L. (2001). Análise de comportamentos de crianças expostas à punção venosa para quimioterapia (Tese de doutorado). Universidade de Brasília, Brasília.

Crepaldi, M. A., Rabuske, M. M., \& Gabarra, L. M. (2006). Modalidades de atuação do psicólogo em Psicologia Pediátrica. In M. A. Crepaldi, M. B. M. Linhares, \& G.B.
Perosa (Eds.). Temas em psicologia pediátrica (pp. 1355). São Paulo: Casa do Psicólogo.

Dolgin, M. J., Phipps, S., Fairclough, D. L., Sahler, O. J. Z., Askins, M., Noll, R. B., ... Katz, E. R. (2007). Trajectories of adjustment in mothers of children with newly diagnosed cancer: A natural history investigation. Journal of Pediatric Psychology, 32(7), 771-782.

Duarte, I. B. (2012). Efeito de instruções e de treino parental sobre comportamentos observados em cuidadores $e$ em crianças com câncer durante sessão de punção venosa para quimioterapia ambulatorial (Dissertação de mestrado). Universidade Federal do Pará, Belém.

Echer, I. C. (2005). Elaboração de manuais de orientação para o cuidado em saúde. Revista Latino-Americana de Enfermagem, 13(5), 754-757.

Ferreira, E. A. P. (2006). Adesão ao tratamento em psicologia pediátrica. In M. A. Crepaldi; M. B. Linhares \& G. B. Perosa (Eds.). Temas em Psicologia Pediátrica (pp. 147-189). São Paulo: Casa do Psicólogo.

Ferreira, R. S. (2005). Efeitos da apresentação sistematizada de um manual educativo para pais de crianças com leucemia (Dissertação de mestrado). Universidade de Brasília, Brasília.

Fonseca, L. M. M., Scochi, C. G. S., Rocha, S. M. M., \& Leite, A. M. (2004). Cartilha educativa para orientação materna sobre os cuidados com o bebê prematuro. Revista Latino-Americana de Enfermagem, 12(1), 65-75.

Gomes, D. L. (2010). Efeitos da automonitoração sobre comportamento de adesão a dois tipos de regras nutricionais em adultos com diabetes Tipo 2 (Dissertação de mestrado). Universidade Federal do Pará, Belém.

Grippo, M., \& Fracolli, L. (2008). Avaliação de uma cartilha educativa de promoção ao cuidado de criança a partir da percepção da família sobre temas de saúde e cidadania. Revista da Escola de Enfermagem - USP, 42(3), 430-436.

Hoekstra-Weebers, J. E., Heuvel, F., Jaspers, J. P., Kamps W. A., \& Klip, E. C. (1998). Brief report: An intervention program for parents of pediatric cancer patients: a randomized controlled. Journal of Pediatric Psychology, 23(3), 207-214.

Jonas, A. L. (1997). O que é auto-regra? In R. A. Banaco (Ed.). Sobre comportamento e cognição: Vol. 1. Aspectos teóricos, metodológicos e de formação em análise do comportamento e terapia cognitivista (pp. 144-147). São Paulo: Arbytes.

Kohlsdorf, M., \& Costa Jr., A. L. (2008). Estratégias de enfrentamento de pais de crianças em tratamento de câncer. Estudos de Psicologia (Campinas), 25(3), 417-429.

Kohlsdorf, M., \& Costa Jr., A. L. (2009). Enfrentamento entre cuidadores de pacientes pediátricos em tratamento de leucemia. Interação em Psicologia, 13(2), 263-274. 
Lemos, I. P., \& Ferreira, E. A. P. (2010). Comportamento de crianças, acompanhantes e auxiliares de enfermagem durante sessão de punção venosa. Psicologia: Teoria e Pesquisa, 26(2), 433-443.

Lima, M. B. S. (2009). O uso de manual como estratégia para a redução de ansiedade de acompanhantes de crianças submetidas à cirurgia de especialidade otorrinolaringológica (monografia). Universidade Federal do Pará, Belém.

MacFarlane, J. T., Holmes, W. F, \& MacFarlane, R. M. (1997). Reducing reconsultations for acute lower respiratory tract illness with an information leaflet: A randomized controlled study of patients in primary care. British Journal of General Practice, 47(1), 719-722.

McPherson, C. J., Higginson, I. J., \& Hearn, J. (2001). Effective methods of giving information on cancer: A systematic literature review of randomized controlled trials. Journal of Public Health Medicine, 23(3), 227234.

Moreira, M. F., Nóbrega, M. M. L., \& Silva, M. I. T. (2003). Comunicação escrita: contribuição para a elaboração de material educativo em saúde. Revista Brasileira de Enfermagem, 56(2), 194-198.

Oliveira, M. S., Fernandes, A. F. C., \& Sawada, N. O. (2008). Manual educativo para o autocuidado da mulher: Um estudo de validação. Texto \& Contexto Enfermagem, 17(1), 115-123.

Pierce, L. L. (2010). How to choose and develop written educational materials. Rehabilitation Nursing, 35(3), 99112.
Reberte, L. M., Hoga, L. A. K., \& Gomes, A. L. Z. (2012). $\mathrm{O}$ processo de construção de material educativo para a promoção da saúde da gestante. Revista LatinoAmericana de Enfermagem, 20(1), 1-8.

Salles, P. S., \& Castro, R. C. B. R. (2010). Validação de material informativo a pacientes em tratamento quimioterápico e aos seus familiares. Revista da Escola de Enfermagem USP, 44(1), 182-189.

Silva, G. R. F., \& Cardoso, M. V. L. M. L. (2009). Percepção de mães sobre um manual educativo sobre estimulação visual da criança. Revista Eletrônica de Enfermagem, 11(4), 847-857.

Syrjala, K. L., Abrams, J. R., Polissar, N. L., Hansberry, J., Robison, J., DuPen, S., ... DuPen, A. (2008). Patient training in cancer pain management using integrated print and video materials: A multisite randomized controlled trial. Pain, 135(1-2), 175-86.

Torres, H., Candido, N., Alexandre, L., \& Pereira, F. (2009). O processo de elaboração de cartilhas para orientação do autocuidado no programa educativo em diabetes. Revista Brasileira de Enfermagem, 62(2), 312-316.

Vasconcellos-Silva, P. R., Rivera, F. J. U., \& Rozemberg, B. (2003). Próteses de comunicação e alinhamento comportamental sobre impressos hospitalares. Revista Saúde Pública, 37(4), 531-542.

Agradecemos à equipe de profissionais do Hospital Ophir Loyola pelo apoio à realização desta pesquisa e à Dra. Hilma Tereza Tôrres Khoury, pelo auxílio na análise dos dados.

Nota:

1 Pesquisa parcialmente financiada pelo CNPq, por meio de bolsa de iniciação científica (PIBIC/CNPq/UFPA) para a primeira autora. 